\title{
Physical properties of IP Pegasi: an eclipsing dwarf nova with an unusually cool white dwarf
}

\section{C.M. Copperwheat}

Department of Physics, University of Warwick, Coventry, CV4 7AL, UK

E-mail: c.copperwheat@warwick.ac.uk

\section{T.R. Marsh ${ }^{1}$, V.S. Dhillon ${ }^{2}$, S.P. Littlefair ${ }^{2}$, R. Hickman ${ }^{1}$, B.T. Gänsicke ${ }^{1}$ and}

\section{J. Southworth ${ }^{3}$}

${ }^{1}$ Department of Physics, University of Warwick, Coventry, CV4 7AL, UK

${ }^{2}$ Department of Physics and Astronomy, University of Sheffield, S3 7RH, UK

${ }^{3}$ Astrophysics Group, Keele University, Newcastle-under-Lyme, ST5 5BG, UK

\begin{abstract}
We present high speed photometric observations of the eclipsing dwarf nova IP Peg taken with the triple-beam camera ULTRACAM mounted on the William Herschel Telescope. We obtained eighteen observations of the eclipse in 2004/2005, simultaneous in the Sloan $u^{\prime}, g^{\prime}$ and $r^{\prime}$ bands. By phase-folding and averaging our data we make the first significant detection of the white dwarf ingress in this system and thus make the most secure photometric determinations of the binary parameters in this system to date, which are in agreement with the best spectroscopic determinations. The white dwarf temperature is more difficult to determine, since the white dwarf is seen to vary significantly in flux, even between consecutive eclipses. This is seen particularly in the $u^{\prime}$-band, and is probably the result of absorption by disc material. Our best estimate of the temperature is $10,000-15,000 \mathrm{~K}$, which is much lower than would be expected for a CV with this period, and implies a mean accretion rate that is more than 40 times lower than the expected rate. Unless the mass transfer in IP Peg has begun very recently, these findings imply either that CVs can sustain accretion rates well below the expected rate for very long periods of time, or that the classical picture whereby the long-term accretion rate scales with period is not correct.
\end{abstract}

High Time Resolution Astrophysics IV - The Era of Extremely Large Telescopes - HTRA-IV,

May 5-7, 2010

Agios Nikolaos, Crete, Greece

\footnotetext{
*Speaker.
} 


\section{Introduction}

Cataclysmic variable stars (CVs: [11]) provide examples of white dwarfs accreting from low mass companions at rates of $\sim 10^{-11}-10^{-9} M_{\odot} / \mathrm{yr}$. The mass transfer in CVs is due to angular momentum loss, which is thought to be driven by gravitational radiation at shorter periods and magnetically-coupled stellar winds at longer ( $\sim 3 \mathrm{~h}$ or greater) periods. While this is observationally supported to some extent [7], there is no one-to-one relationship between period and mass transfer rate $(\dot{M})$. The most likely explanation is that the instantaneous $\dot{M}$ we measure is a poor indicator of the mean $\dot{M}$, so that even high mean $\dot{M}$ systems spend some fraction of their time as slowly accreting dwarf novae.

One independent means of inferring a longer-term $\left(10^{3}-10^{5}\right.$ yr $)$ value of $\dot{M}$ is through measurement of the white dwarf temperature. The temperature is a good tracer of the long-term $\dot{M}$ since it is determined not by the accretion heating, but by the compression of the underlying white dwarf by the accreted matter $[9,10]$. Spectroscopically determined white dwarf temperatures are likely to be subject to an observational bias, and so we observed the $3.8 \mathrm{~h}$ period eclipsing dwarf nova IP Pegasi (IP Peg hereafter) with the aim of making an (unbiased) photometric measurement of the white dwarf temperature.

\section{Observations}

The high speed CCD camera ULTRACAM [4] was mounted on the 4.2m William Herschel Telescope (WHT) in August 2005. Observations of IP Peg were made with this instrument between 9 - 15 August, and 25 August - 1 September. Over these periods, 16 separate observations of the IP Peg eclipse were made. In addition, two observations of the eclipse were made in August 2004. ULTRACAM is a triple beam camera and all observations were made using the SDSS $u^{\prime}, g^{\prime}$ and $r^{\prime}$ filters, except for the first 2004 observation, in which the $i^{\prime}$ filter was used in place of $r^{\prime}$, for scheduling reasons. All of these data were reduced with aperture photometry using the ULTRACAM pipeline software, with debiassing, flatfielding and sky background subtraction performed in the standard way. A nearby comparison star was used to compensate for variations in observing conditions, and observations of standard stars [8] taken in evening twilight were used to fluxcalibrate the data. Typical exposure times were $1.5 \mathrm{~s}$

\section{Results}

The light curves show one obvious single ingress feature, followed by separate egresses of the white dwarf, and later, the bright spot. We combined and phase-folded our data and fitted a model to them using the MCMC method. The model consisted of a white dwarf primary, a Roche lobe filling secondary and accretion disc and bright spot components, and is described in detail in [3]. We plot the phase-folded light curves and model fits in Figure 1. In these phase folded light curves we detect the white dwarf ingress feature, which is separate from and prior to the much deeper bright spot ingress. This is the first time the white dwarf ingress has been identified: in the individual light curves it is obscured by the accretion driven flickering.

One complication is that previous authors have suggested the white dwarf egress to be variable in both size and duration $[15,16]$. Consequently after determining the system parameters by fitting 


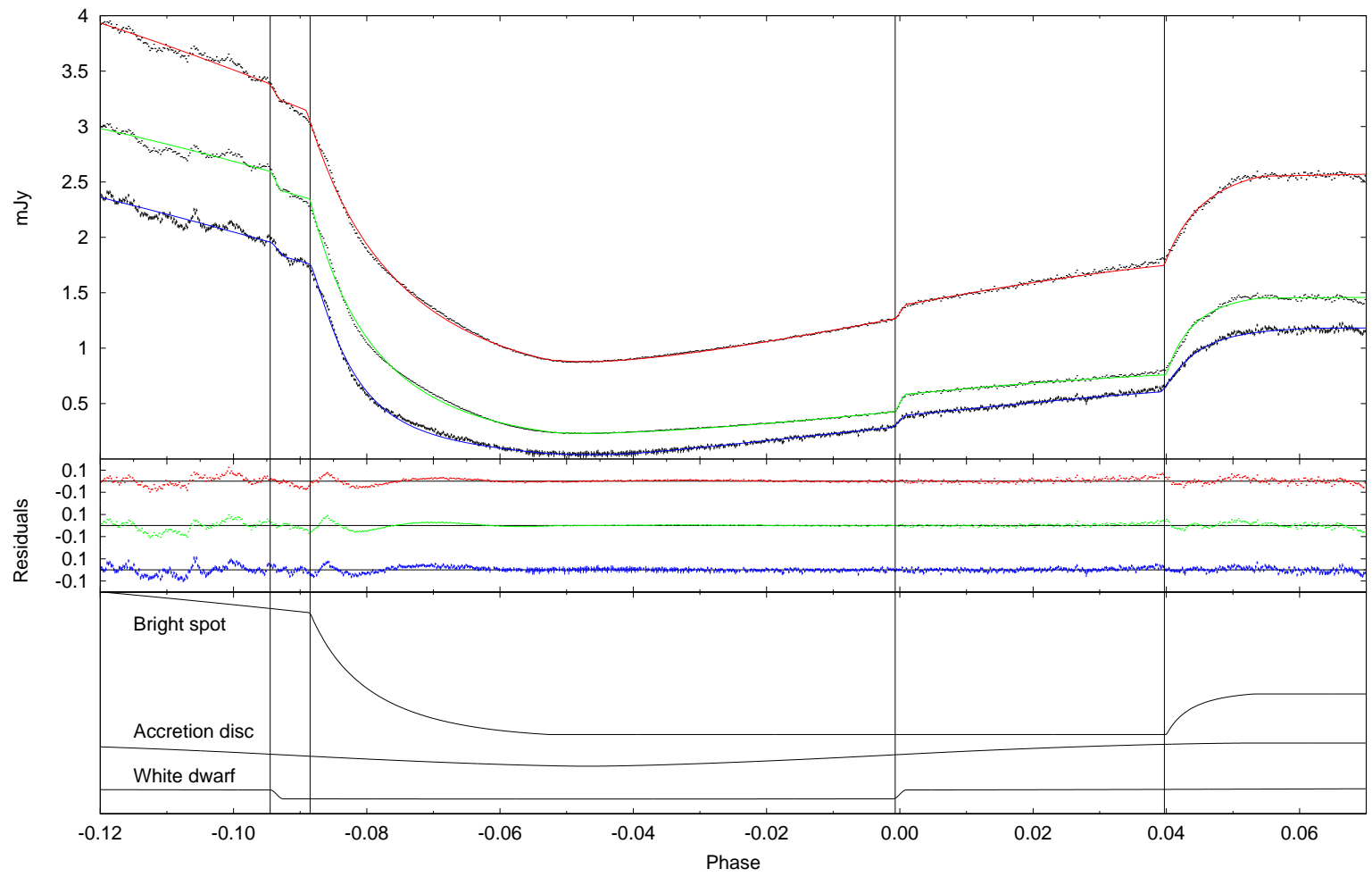

Figure 1: Top panel: phase-folded and binned light curves (top, $r^{\prime}$; middle, $g^{\prime}$; bottom, $u^{\prime}$ ), comprised of the eleven complete eclipse observations made in 2004 and 2005. We plot the average flux in mJy against the binary phase, where a phase of 0 corresponds to the mid-egress of the white dwarf. We plot the datapoints with uncertainties in black, and the best model fits to these data in red, green and blue (for $r^{\prime}, g^{\prime}$ and $u^{\prime}$, respectively). The four vertical lines indicate the start of (from left to right) the white dwarf ingress, the bright spot ingress, the white dwarf egress and the bright spot egress. Middle panel: residuals in the three bands (top, $r^{\prime}$; middle, $g^{\prime}$; bottom, $u^{\prime}$ ). Bottom panel: the three components of the $g^{\prime}$-band model plotted separately, showing the relative strengths of the bright spot, accretion disc and white dwarf. These three lines have been offset for clarity.

the phase-folded light curves, we refitted each light curve individually to determine the white dwarf contribution for each individual light curve. In Figure 2 the $u^{\prime}$-, $g^{\prime}$ - and $r^{\prime}$-band light curves of the region around the white dwarf egress are plotted, with the accretion disc component of the emission subtracted from the data. This plot shows clearly that the height of the egress feature and hence the white dwarf contribution does vary significantly from eclipse to eclipse.

\section{Discussion}

The phase width of the white dwarf eclipse is an observable quantity that is intrinsically linked to two physical properties: the mass ratio and the binary inclination [1]. With the additional geometric information resulting from measurement of the bright spot ingress and egress phases the mass ratio and inclination in this system can be determined photometrically. Since we observe for the first time the white dwarf ingress, we make the first accurate measurement of the phase 


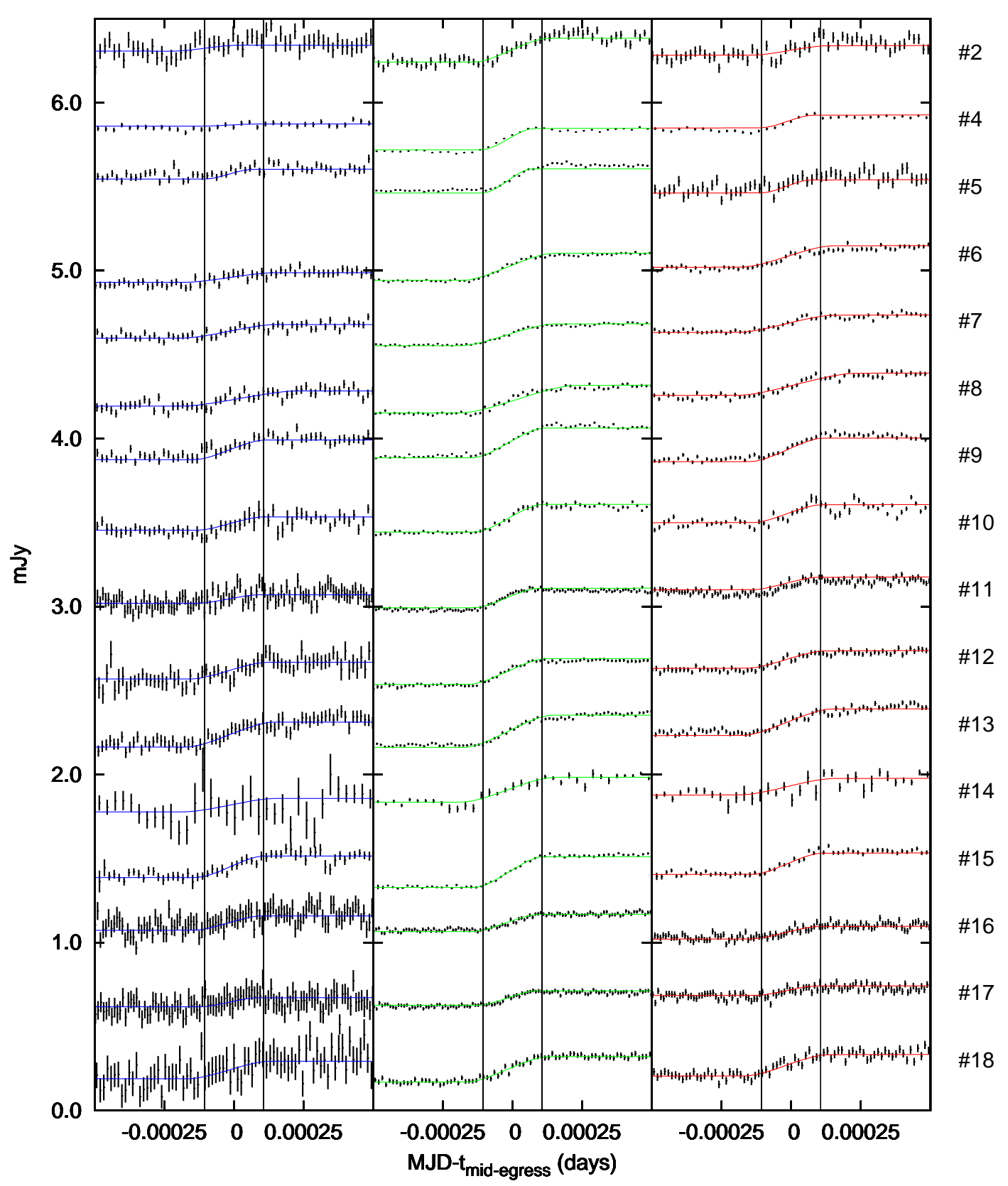

Figure 2: The region of the light curves around the white dwarf egress for sixteen of the eighteen eclipses. We plot the $u^{\prime}$-band data in the first column, the $g^{\prime}$-band data in the second and the $r^{\prime}$-band data in the third. The vertical lines identify the mean phase width of the white dwarf egress. 

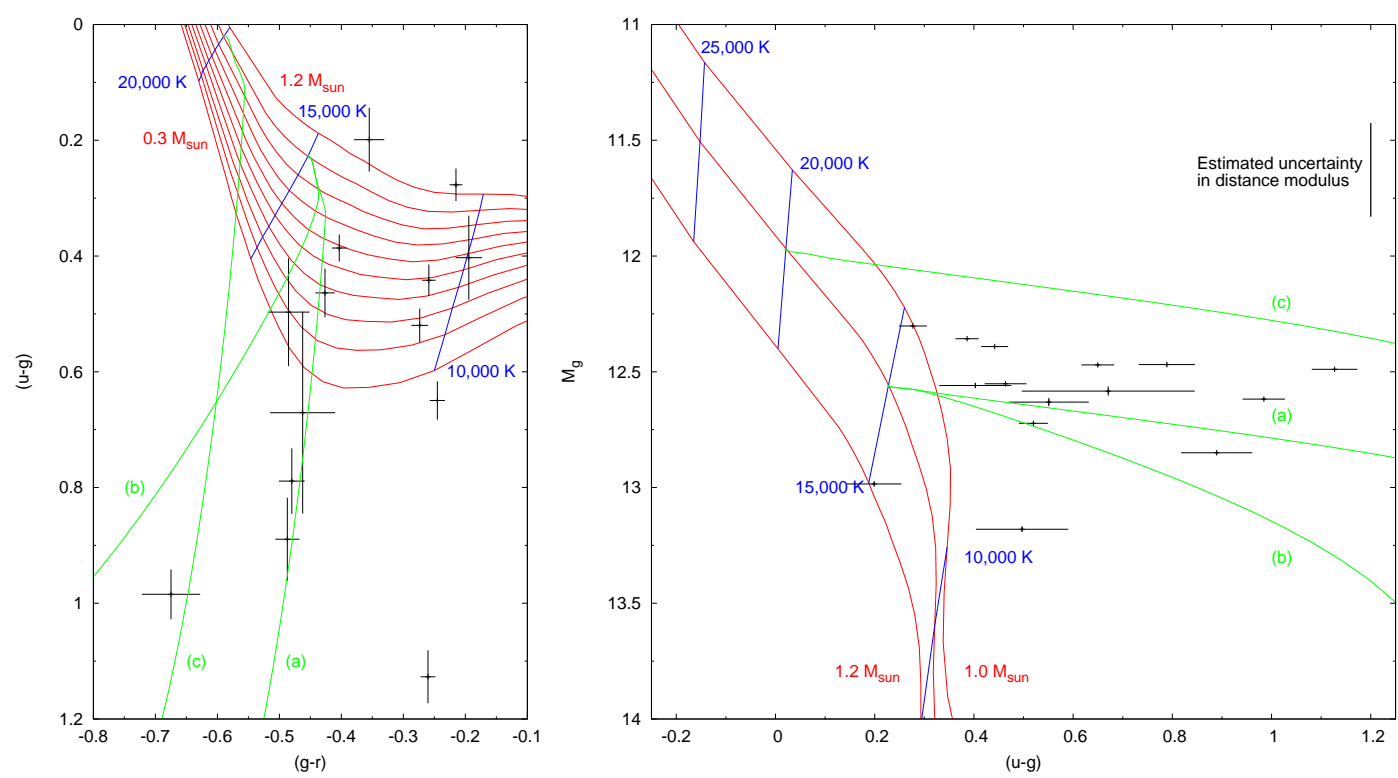

Figure 3: We plot the colours and magnitude of the white dwarf for each individual eclipse. In the left panel we plot the $u^{\prime}-g^{\prime}$ and $g^{\prime}-r^{\prime}$ colours for the white dwarf, and in the right panel we show the $u^{\prime}-g^{\prime}$ colour versus the absolute magnitude $M_{g^{\prime}}$. For this we indicate to the right of the plot our estimate of the uncertainty in the distance modulus. The red lines that run from the top left area of each panel are synthetic white dwarf tracks [5]. In the left panel we plot tracks for a white dwarf mass of 0.3 to $1.2 M_{\odot}$, plotted every $0.1 M_{\odot}$, but for clarity in the right panel we just show the tracks for a mass of 1.0,1.1 and $1.2 M_{\odot}$. Perpendicular to these tracks we plot isotherms (blue) every $5,000 \mathrm{~K}$. The green lines are example tracks for a simple photoelectric absorption model which is consistent with the variations in white dwarf colour which we observe [3].

width of the white dwarf eclipse, which we find to be $\Delta \phi=0.0935 \pm 0.0003$. This is higher than the previous best value of 0.0863 [15]. We subsequently find the mass ratio in the $g^{\prime}$-band data to be $q=0.48 \pm 0.01$ and the inclination to be $83.81 \pm 0.45$. This inclination is higher than most previous determinations, with the difficulty in identifying the white dwarf ingress feature, and the subsequent under-estimation of the phase width of the white dwarf's eclipse, being the confounding factor.

We found the white dwarf mass to be $1.16 \pm 0.02 M_{\odot}$, in close agreement with the best spectroscopic determination [12]. The donor mass and radius were found to be $0.55 \pm 0.02 M_{\odot}$ and $0.47 \pm 0.01 R_{\odot}$ respectively. This is low given our mass determination, suggesting a donor that is slightly undersized compared to an equivalent main sequence star [2]. This is unusual for an accreting binary: donor stars tend to be oversized, due to either evolution or being out of thermal equilibrium as a result of mass loss. Our finding is consistent with a donor in thermal equilibrium.

We see in Figure 2 that, while the length of the white dwarf egress appears constant, its flux varies significantly on timescales of days. In the left panel of Figure 3 we plot the $u^{\prime}-g^{\prime}$ and $g^{\prime}-r^{\prime}$ colours of the white dwarf. We plot also synthetic white dwarf tracks [5] for a white dwarf mass range of 0.3 to $1.2 M_{\odot}$. In the right panel we plot $u^{\prime}-g^{\prime}$ against the absolute $g^{\prime}$-band magnitude $M_{g}^{\prime}$. We assume a distance of $151 \mathrm{pc}$ [3]. This figure shows apparent variations in the white dwarf 
colour, particularly in $u^{\prime}-g^{\prime}$. It is most likely that this variation is due to photoelectric absorption, as a result of obscuration of the white dwarf by material from the accretion disc. The green tracks in Figure 3 are the results of a simple absorption model [3] and seem consistent with the observed behaviour. An occulting region of disc material could explain the past reports of very extended white dwarf egresses in this system [15]. Alternatively these might have been failures to detect the variable egress at all.

By comparing the data points with the synthetic tracks, Figure 3 suggests a white dwarf temperature of between 10,000 to $15,000 \mathrm{~K}$; it is difficult to be more precise than this due to the flux variations. We assume that our points with the bluest $u^{\prime}-g^{\prime}$ colour are indicative of a white dwarf that is largely unobscured. This temperature range is remarkably cool. Previous studies have suggested that a white dwarf in a $\mathrm{CV}$ with a period $>3 \mathrm{~h}$ would have a typical temperature of between $25,000-35,000 \mathrm{~K}[14,9]$. Such a temperature is clearly inconsistent with our IP Peg data. If we examine this result in the context of the medium term $\left(10^{3}-10^{5} \mathrm{yr}\right)$ accretion rate, our value for the IP Peg white dwarf implies a mean accretion rate of $<5 \times 10^{-11} M_{\odot} \mathrm{yr}^{-1}$. The expected rate for a dwarf nova of this period is $\sim 2 \times 10^{-9} M_{\odot} \mathrm{yr}^{-1}$ : more than 40 times greater. The value we find is much closer to the instantaneous rate. This low mass transfer rate may also explain why we find the donor to be in thermal equilibrium.

The low temperature of this system suggests there could be a selection bias in the existing sample of long-period systems with spectroscopically determined temperatures [9], and further detailed studies of eclipsing systems are necessary in order to determine the extent of this bias. At this stage, for example, it is possible that mass transfer in IP Peg has begun very recently and so the temperature in this system is a poor indicator of the long term rate. Alternatively, we can take our low value for the mass transfer rate over the past $10^{3}-10^{4} \mathrm{yr}$ in this system to be correct, which would suggest it has sustained an accretion rate well below the expected mean rate for more than 1000 years. If all 'steady-state' CVs go through low accretion rate dwarf nova phases, then this result implies the length of these phases can be very long indeed. The third possibility is that there are problems with the picture of $\mathrm{CV}$ evolution as driven by angular momentum loss, and alternatives to the classical disrupted magnetic braking model (such as [13]) should be explored.

\section{References}

[1] Bailey J., The dwarf nova Z Chamaeleontis. I - Photometry, MNRAS, 1979, 187, 645

[2] Baraffe I., Chabrier G., Allard F., Hauschildt P. H., Evolutionary models for solar metallicity low-mass stars: mass-magnitude relationships and color-magnitude diagrams, AAP, 1998, 337, 403

[3] Copperwheat C. M., Marsh T. R., Dhillon V. S., Littlefair S. P., Hickman R., Gänsicke B. T., Southworth J., Physical properties of IP Pegasi: an eclipsing dwarf nova with an unusually cool white dwarf, MNRAS, 2010, 402, 1824

[4] Dhillon V. S.. Marsh T. R., Stevenson M. J., Atkinson D. C., Kerry P., Peacocke P. T., Vick A. J. A., Beard S. M., Ives D. J., Lunney D. W., McLay S. A., Tierney C. J., Kelly J., Littlefair S. P., Nicholson R., Pashley R., Harlaftis E. T., O'Brien K., ULTRACAM: an ultrafast, triple-beam CCD camera for high-speed astrophysics, MNRAS, 2007, 378, 825

[5] Holberg J. B. and Bergeron P., Calibration of Synthetic Photometry Using DA White Dwarfs, AJ, 2006, 132, 1221 
[6] Ritter H. and Kolb U., Catalogue of cataclysmic binaries, low-mass X-ray binaries and related objects (Seventh edition), AAP, 2003, 404, 301

[7] Shafter A. W., The role of the dwarf nova period distribution in understanding the evolution of cataclysmic variables, ApJ, 1992, 394, 268

[8] Smith J. A., Tucker D. L., Kent S., Richmond M. W., Fukugita M., Ichikawa T., Ichikawa S.-i., Jorgensen A. M., Uomoto A., Gunn J. E., Hamabe M., Watanabe M., Tolea A., Henden A., Annis J., The u'g'r'i'z' Standard-Star System, AJ, 2002, 123, 2121

[9] Townsley D. M. and Bildsten L., Measuring White Dwarf Accretion Rates via Their Effective Temperatures, ApJL, 2003, 594, L227

[10] Townsley D. M. and Gänsicke B. T., Cataclysmic Variable Primary Effective Temperatures: Constraints on Binary Angular Momentum Loss, ApJ, 2009, 693, 1007

[11] Warner B., Cataclysmic variable stars, Cambridge University Press, Cambridge, 1995

[12] Watson C. A., Dhillon V. S., Rutten R. G. M., Schwope A. D., Roche tomography of cataclysmic variables - II. Images of the secondary stars in AM Her, QQ Vul, IP Peg and HU Aqr, MNRAS, 2003, 341,129

[13] Willems B., Taam R. E., Kolb U., Dubus G., Sandquist E. L, Theoretical Orbital Period Distributions of Cataclysmic Variables above the Period Gap: Effects of Circumbinary Disks, ApJ, 2007, 657, 465

[14] Winter L. and Sion E. M., Composite Accretion Disk and White Dwarf Model Analyses of the Quiescence of Dwarf Novae: EM Cygni, CZ Orionis, and WW Ceti, ApJ, 2003, 582, 352

[15] Wood J. and Crawford C. S., An estimate of the system parameters in the dwarf nova IP Peg, MNRAS, 1986, 222, 645

[16] Wood J. H., Marsh T. R., Robinson E. L., Stiening R. F., Horne K., Stover R. J., Schoembs R., Allen S. L., Bond H. E., Jones D. H. P., Grauer A. D., Ciardullo R., The ephemeris and variations of the accretion disc radius in IP Pegasi, MNRAS, 1989, 239, 809 